\title{
Assessment of Demographic Profile and Cytopathological Diagnosis of Thoracic Mass Lesions Using CT-guided FNAC
}

\author{
Dimple Darad and Savitri Chauhan*
}

Department of Pathology, GMERS Medical College, Gotri, Vadodara, Gujarat, India

\section{ABSTRACT}

Background and Aim: Computed tomography (CT) guided Fine needle aspiration cytology (FNAC) is a simple, safer, and rapid technique. CT guidance can assist FNAC from any part of thorax without any major complications. FNAC helps distinguish between benign and malignant lesions and also effectively aids in morphological typing of lung cancer, thereby reducing the delay in initiation of specific therapy like chemotherapy or surgery.

Materials and Method: Present study was carried out at a department of pathology GMERS Medical College, Gotri, Vadodara, Gujarat. One hundred and twenty three cases of lung mass lesions were evaluated with CT guided FNAC. Smears were stained with May-Grunwald Giemsa and Hemotoxylin \& Eosin (H \& E) stain for rapid cytopathological evaluation of the lesions.

Results: Out of 123 cases included in the study, 90 cases (73.17\%) were male and $33(26.83 \%)$ were female. The mean age in malignant cases was 60.3 years whereas it was 50 years in benign lesions. Cytological examination showed that 109 cases were malignant and 14 cases were benign lesions. Cytomorphological diagnosis revealed maximum number of cases belonged to adenocarcinoma (60.9\%). Rest of the cases were Squamous cell carcinoma (16.2\%), Non-specific inflammation (4.8\%), Granulomatous inflammation (3.2\%), metastatic deposits (3.2\%) and spindle cell neoplasm (1\%). Only ten cases could be followed up for histopathological evaluation. All the cases confirmed the primary diagnosis of malignancy on histopathological examination.

Conclusion: CT guided FNAC is a simple, and reliable for the diagnosis and sub typing of lung cancer, thereby reducing the delay in initiation of specific therapy like chemotherapy or surgery.

\section{Keywords: Adenocarcinoma, Computed Tomography, Fine Needle Aspiration Cytology, Squamous Cell Carcinoma}

\section{Introduction}

Cytological evaluation of lung mass lesions can be reliably done by percutaneous transthoracic fine needle aspiration cytology. ${ }^{[1]}$ Though Leyden (1883) and in 1986, Menbriel introduced the procedure as diagnostic lung puncture for detection of infections and malignancy, only in recent few years the CT guided FNAC has gained momentum and acceptance for its diagnostic usefulness of clinical cytology in lung and mediastinal cancer diagnosis. ${ }^{[2]}$ FNAC is a simple, safer, and rapid technique, particularly with the aid of computed tomography (CT) scan. CT guidance can assist FNAC from any part of thorax without any major complications since the needle path avoiding major blood vessels can be planned. ${ }^{[3]}$ FNAC helps distinguish between benign and malignant lesions and also effectively aids in morphological typing of lung cancer, thereby reducing the delay in initiation of specific therapy like chemotherapy or surgery. Literature demonstrates that CT-guided FNAC is an sensitive and accurate method for diagnosis of lungs malignancy. Pneumothorax, minor bleeding, chest pain and hemoptysis are some the commonly seen complications of this technique. However, only a few require active management. ${ }^{[4]}$ Patients with severe emphysema, bleeding diathesis, inability to hold breath, uncontrolled cough, hydatid cyst and arteriovenous aneurysm are some of the contraindications of the technique. ${ }^{[5]}$ The aims of the study was to analyze the age, sex, location of the lung lesions and cytopathological diagnosis of thoracic mass lesions using CT-guided FNAC.

\section{Materials and Method}

Present study was carried out at a department of pathology GMERS Medical College, Gotri, Vadodara, Gujarat. All the consecutive cases of thoracic lesions who were referred for CT guided FNA were registered for the study. 132 patients having lung lesions suspicious of malignancy by chest radiography and/or CT scan were referred from departments like Pulmonology, General medicine and surgical oncology. Out of 132 cases, only 123 cases could be included for the study, as cytological examination showed an inadequate cell yield in nine cases. Informed consent was taken from each patient. The exact location of lung mass, size, distance of the lesion from skin, site, angle of entry and route of the needle was ascertained after taking measurements on the CT scan monitor. Under all 
aseptic precautions, a long spinal needle $(21 \mathrm{G}-88 \mathrm{~mm})$ was introduced percutaneously. A CT scan slice was taken, after introducing the spinal needle, to confirm the presence of needle tip within the mass. The aspirates were obtained by to and fro movements of the needle within the lesions and five to six smears were immediately prepared from the aspirate in the CT scan room. Air-dried smears were stained with May-Grunwald Giemsa stain and alcoholfixed smears were stained with H\&E stain for rapid cytopathological evaluation of the lesions. Patients were monitored for two hours to check for development of any complications. Patients having severe Chronic obstructive pulmonary disease (COPD), bleeding disorders and pulmonary arterial hypertension (PAH) were not taken for CT guided FNA. Subsequent biopsy specimens which were received for histopathological examination (HPE) were stained with routine Hematoxylin and Eosin $(\mathrm{H}$ and E) stain and examined under light microscope. Smears were studied and classified into three groups as follows: Positive for malignancy and morphologicall diagnosis wherever possible. Suspicious for malignancy with smears having 2 atypical cells with no definite evidences of malignancy or cellularity too less to opine definitively. Negative for malignancy or other inflammatory lesions. All the morphologically diagnosed cases were further classified as small cell lung carcinoma (SCLC), nonsmall cell lung carcinoma (NSCLC), metastatic lesions and benign lesions. Specific infections were reported as benign lesions. Further classification of NSCLCs cases as Squamous cell carcinoma (SCC), Adenocarcinoma (ADC), and undifferentiated large cell carcinoma (Undiff. LCC) according to cytomorphology of malignant cells was done wherever possible.

\section{Results}

Out of 123 cases included in the study, 90 cases $(73.17 \%)$ were male and $33(26.83 \%)$ were female. Cytological examination showed that 109 cases were malignant and 14 cases were benign lesions. Cytomorphological diagnosis revealed maximum number of cases belonged to Table 1: Demographic features and distribution of site of the lesion. adenocarcinoma (60.9\%). Rests of the cases were Squamous cell carcinoma (16.2\%), Non-specific inflammation (4.8\%), Granulomatous inflammation (3.2\%), metastatic deposits $(3.2 \%)$ and spindle cell neoplasm (1\%). A case each of melanoma and lymphoma were identified. A case each of Candidiasis, Cryptococcosis, Nocardia and Mucormycosis were also noted. The mean age in malignant cases was 60.3 years whereas it was 50 years in benign lesions. There were three cases under the age of 40 years with youngest being 32 years who was diagnosed to have Adenocarcinoma. Fifth and sixth decade group had majority of the cases. History of chronic smoking was present in $57 \%$ of the patients. The general demographic findings are tabulated in Table 1.

In the present study, a total of 106 malignant cases were detected on FNAC. Among the malignant lesions, adenocarcinoma was the most common $(60.9 \%)$ followed by squamous cell carcinoma (16.2\%). Male preponderance in both adenocarcinoma $(2: 1)$ and squamous cell carcinoma (5:1) was noted.

FNAC revealed, 109 cases were malignant and 14 cases were benign. Only ten cases could be followed up for Histopathological evaluation. All the cases confirmed the primary diagnosis of malignancy on histopathological examination. Two cases which were diagnosed to be suspicious of malignancy and advised core needle biopsy were confirmed to be squamous cell carcinoma with intense inflammatory reaction on histopathology. In cartain cases like carcinoid tumour and spindle cell lesions, Immunohistochemistry (IHC) markers like chromogranin A, cytokeratin, and S-100 were performed for confirmation of the diagnosis.

Microscopically, classical cytomorphological features were observed in most of the adenocarcinomas, squamous cell carcinomas and small cell carcinomas. In the present study, we found a case of Melanoma, one case of bronchioloalveolar carcinoma, two cases of thymoma and one case of atypical spindle cell neoplasm.

\begin{tabular}{|l|c|c|}
\hline Variable & Subcategory & Number \\
\hline \multirow{4}{*}{ Age } & & 3 \\
\cline { 2 - 3 } & $20-30$ & 11 \\
\cline { 2 - 3 } & $31-40$ & 20 \\
\cline { 2 - 3 } & $41-50$ & 30 \\
\cline { 2 - 3 } & $51-60$ & 19 \\
\cline { 2 - 3 } & $61-70$ & 18 \\
\cline { 2 - 3 } & $71-80$ & 2 \\
\cline { 2 - 3 } & $>80$ & 20 \\
\hline
\end{tabular}




\begin{tabular}{|c|c|c|}
\hline Variable & Subcategory & Number \\
\hline \multirow{2}{*}{ Gender } & & 90 \\
\hline \multirow{2}{*}{ Location } & Male & 33 \\
\hline & Female & 85 \\
\cline { 2 - 3 } & & 36 \\
\cline { 2 - 3 } & Left Lung & 2 \\
\cline { 2 - 3 } & Right Lung & \\
\cline { 2 - 3 } & Mediastinum & \\
\hline
\end{tabular}

\section{Discussion}

FNAC is a simple, minimally invasive and relatively safer invasive procedure for evaluation of pulmonary lesions. It enables fairly accurate stratification of malignant tumours in majority of cases. In our study, cytological evaluation of 123 pulmonary mass lesions was carried out over a period of two years. Adequate cellularity for cytomorphological diagnosis was obtained by CT guided FNAC. Fifth and sixth decade recorded the peak incidence which was comparable with other recent studies. [6,7,8,9] Cytomorphological features revealed, out of 123 cases, $3(2.4 \%)$ as benign, $106(86.1 \%)$ as malignant and $14(11.3 \%)$ cases as inflammatory/ infectious origin. The higher incidence of malignant tumours may be because of CT guided FNAC was primarily done for evaluation of lung mass lesions. The mean age in the present study was 58.9 years, which is almost similar to studies conducted by Singh et al.(56.4 years), ${ }^{[10]}$ and and Saha et al., ${ }^{[11]}$ (56.8 years). Power et al., ${ }^{[13]}$ also showed higher prevalence of pulmonary neoplasms after the age of 50 years, with an average age of 54 years. There was male preponderance $(73.1 \%)$ in the present study. The percentage of male patients was comparable in our series with the recent studies i.e., 71.1\%, (15) $78.9 \%$ and $80.6 \%$, respectively. ${ }^{[7]}$ In our study, right sided lung tumors were more common than the left sided lung tumours. The most common site of lung tumors on CT scan findings was right upper zone. Majority of the pulmonary mass lesions were $5-10 \mathrm{~cm}$ in diameter, according to size of the lesions. Size variation among the thoracic mass lesions was more as compared to studies conducted by Shah. S. et.al ${ }^{[14]} 64.7 \%$ were active smokers among the patients in our study. Cytological evaluation was done in 123 out of 132 cases $(95.38 \%)$ and the higher incidence of malignant lesions $(86.1 \%)$ was comparable with that found in other studies. ${ }^{[11,12,15]}$ The malignant lesions formed the major category which included carcinomas, lymphoma, melanoma and metastatic deposits. Among all 106 cases of malignant lung tumors, the most common were adenocarcinomas followed by squamous cell carcinoma ${ }^{[16]}$ and metastatic deposits (3.2\%). Madan et al ${ }^{[9]}$ and Tan et al ${ }^{[15]}$ reported higher incidence of adenocarcinoma than that of squamous cell carcinoma whereas, in some other recent studies, prevalence of squamous cell carcinoma was reported more. ${ }^{[16,17]}$ In Our study we found higher incidence of adenocarcinoma. This can be partly attributed to our inclusion of BAC as adenocarcinoma. There is a conceptual change regarding diagnosis of BAC. Travis et al., in 2011 recommended discontinuing the term 'BAC', rather concluded to use the term adenocarcinoma in situ (AIS) or minimally invasive carcinoma (MIA) for small foci of invasion. However, the terms AIS amd MIA should not be used in cytology and small biopy specimens. The term Adenocarcinoma in situ (AIS) was used for small $(\leq 3 \mathrm{~cm})$ single foci of adenocarcinoma with pure lepidic growth. For small size and morphology with small foci of invasion $\leq 0.5 \mathrm{~cm}$-a new concept of minimally invasive carcinoma (MIA) to be considered ${ }^{[18]}$ Highly accurate cytological typing can be of great importance in cases where confirmatory histology is not available. Most of the cases of lung malignancy were primary while only four cases represented metastatic adenocarcinoma. In our study, chest pain in five cases was noted which were managed conservatively. Pneumothorax was found to be common complication in other studies, but we did not have any case of pneumothorax in the present study. Overall complication rate $(4.06 \%)$ in our study was also much lower than other series where the complication rates varied from 6 to $50 \%$. ${ }^{[7,14]}$ In our study, CT guided FNAC showed almost perfect agreement with histological diagnosis, though the number of cases for histopathological follow up were much less. So FNAC was found to be highly accurate in diagnosis of lung mass as almost similar shown by previous studies. ${ }^{[19]}$ Hence CT guided FNAC diagnosis alone can be used with confidence to select treatment modalities and to avoid unnecessary surgeries in patients with lung mass lesions.

\section{Conclusion}

CT guided FNAC is a safe, simple, and reliable diagnostic method with high accuracy for the diagnosis and sub classification of lung cancer, thereby reducing the delay in initiation of specific therapy like chemotherapy or surgery. 


\section{References}

1. Saha A, Kumar K, Choudhuri MK. Computed tomographyguided fine needle aspiration cytology of thoracic mass lesions: A study of 57 cases. J Cytol. 2009;26:55-9.

2. Mondal SK, Nag D, Das R, Mandal PK, Biswas PK, Osta M. Computed tomogram guided fine-needle aspiration cytology of lung mass with histological correlation: A study in Eastern India. South Asian Journal of Cancer.2013;2(1):14-18.

3. Konjengbam R, Singh NB, Gatphoh SG. Computed tomography guided percutaneous transthoracic fine needle aspiration cytology of pulmonary mass lesions: Two years cross sectional study of 61 cases. J Med Soc. 2014;28:112-60

4. Yadav RK, Sen R, Agarwal S, Aggarwal S. CT-guided FNAC of intrarthoracic mass lesions - a study among 35 patients. J Indian Med Assoc.2010;108:571-4.

5. Jaya Shankar E, Pavani B, Chandra E, Reddy R, Srinivas M, et al. (2010) Computed Tomography Guided Percutaneous Thoracic: Fine Needle Aspiration Cytology in Lung and Mediastinum. J Cytol Histol 1:107. doi:10.4172/21577099.1000107

6. Syed A, Shabab M, Uddin A. Computed tomography guided fine needle aspiration cytology of lung lesions: A study of 162 cases. J Chittagong Med Coll Teach Assoc. 2009;20:50-2.

7. Bandyopadhyay A, Laha R, Das TK, Sen S, Mangal S, Mitra PK. CT guided fine needle aspiration cytology of thoracic mass lesions: A prospective study of immediate cytological evaluation. Indian J Pathol Microbiol. 2007;50:51-5.

8. Shaheen MZ, Sardar K, Murtaza HG, Safdar SS, Hafeez A, Mushtaq MA, et al. CT guided trans-thoracic fine needle aspiration/biopsy of mediastinal and hilar mass lesions. Indian J Pathol Microbiol. 2010;16:4-13.

9. Madan M, Bannur H. Evaluation of fine needle aspiration cytology in the diagnosis of lung lesions. Turk J Pathol. 2010;26:1-6.

10. Singh J P, Garg L, Setia V. Computed tomography $(\mathrm{Ct})$ guided transthoracic needle aspiration cytology in difficult thoracic mass lesions-not approachable by USG. Indian J Radiol Imaging. 2004;14:395-400.

11. Parajuli S, Tuladhar A, Basnet RB. Ultrasound and computed tomography guided fine needle aspiration cytology in diagnosing intra-abdominal and intra-thoracic lesions. J Pathol Nepal 2011;1:17-21.

12. Prashant, Ramachandra C, Pattbhiraman, Raghuram, Satya V, Attili S. Feasibility, safety, and efficacy of the CT guided fine needle aspiration cytology of lung lesions. Indian J Med Paediatr Oncol 2007;28:16-25.

13. Powers CN, Silverman JF, Geisenger KR, Frable WJ. Fine needle aspiration biopsy of the mediastinum a multi institutional analysis. Am J Clin Pathol. 1996;105:168-73.

14. Shah S, Shukla K, Patel P. Role of needle aspiration cytology in diagnosis of lung tumors. A study of 100 cases. Indian J Pathol Microbiol. 2007;50:56-8.

15. Tan KB, Thamboo TP, Wang SC, Nilsson B, Rajwanshi A, Salto-Tellez M. Audit of transthoracic fine needle aspiration of the lung: Cytological sub classification of bronchogenic carcinomas and diagnosis of tuberculosis. Singapore Med J 2002;43:570-5.

16. Salazar AM, Westcott JL. The role of transthoracic needle biopsy for the diagnosis and staging of lung cancer. Clin Chest Med.1993;14:99-110.

17. Gouliamos AD, Giannopoulos DH, Panagi GM, Fletoridis NK, Deligeorgi-Politi HA, Vlahos LJ. Computed tomography-guided fine needle aspiration of peripheral lung opacities: An initial diagnostic procedure? Acta Cytol 2000;44:344-8.

18. Travis WD, Brambilla E, Noguchi M, Nicholson AG, Geisinger $\mathrm{K}$, Yatabe $\mathrm{Y}$, et al. International association for the study of lung cancer/American thoracic society/ European respiratory society international multidisciplinary classification of lung adenocarcinoma. J Thorac Oncol. 2011 Feb;6(2):244-85.

19. Abrari A, Aziz M, Haq R. Cytology of lung tumors - study of 60 cases with emphasis on accuracy and problem areas. J Cytol. 2003;20:79-81.

*Corresponding author:

Dr Savitri Chauhan, MD, Associate Professor, Department of Pathology, GMERS Medical College, Gotri, Vadodara Gujarat(India)- 390021

Phone: +91 9898067478

Email: savitri_chauhan@yahoo.com

Financial or other Competing Interests: None. 